\title{
A dynamical definition of quasi-bound molecular clusters
}

\author{
Sarah A. Harris and Ian J. Ford \\ Department of Physics and Astronomy, University College London, \\ Gower Street, London WC1E 6BT, United Kingdom.
}

\begin{abstract}
Molecular configurations which count as snapshots of a quasi-bound cluster are identified through a retrospective dynamical definition. The trajectory of a molecular cluster is followed, and a clear evaporation event is considered to have occurred when a molecule moves a very long distance away from the others. The cluster is judged to have broken before this condition is satisfied, however, at the instant that the energy of the departing molecule in the centre of mass frame becomes positive. The decay of a cluster is therefore defined dynamically as the production of a molecule with positive energy on a separating trajectory. Not all positive energy molecules created by the system follow such a trajectory, hence the need to examine the subsequent behaviour in molecular dynamics. We simulate a sequence of decays by repairing broken clusters as they occur. This approach enables us to calculate mean decay rates of isolated Lennard-Jones clusters in what promises to be a physically realistic fashion.
\end{abstract}

PACS numbers:

\section{INTRODUCTION}

The traditional division of substances into gases, liquids and solids appears to miss out an important example of condensed matter: the molecular cluster. Into which category should we place a quasi-bound assemblage of just a few tens of molecules? Clusters can, of course, be viewed quite simply as fragments of liquids or solids, but clusters are also found in gases, where they are responsible, in part, for deviations from ideal gas properties.

Plainly the traditional divisions fail when we study substances at the microscopic level. We must recognise that gases, liquids and solids are defined in continuum thermodynamics as bulk phases which are stable with respect to one another under certain conditions, and which can also coexist, as long as surface effects are neglected. Molecular clusters clearly do not fit into these categories, since they are small and because their interface with their surroundings cannot be neglected. It is possible to extend thermodynamics to small systems [1], but we then encounter a further difficulty: with few exceptions, molecular clusters never coexist with a bulk phase, unless constrained in some way.

We can see this by using continuum thermodynamics to describe a spherical droplet surrounded by a vapour in a closed system. Let us start by fixing the mass and density of the droplet, and allowing the system to relax to thermal equilibrium. In effect, we use the constrained droplet mass to characterise a new 'droplet' phase, in the same way that bulk phases are defined by their density, symmetry, etc. Reiss has emphasised the importance of constraints in thermodynamics [2] and how they often appear implicitly. A free energy of the system can then be computed, comprising bulk contributions from the separate phases, together with a surface term. Now, if the vapour is undersaturated, then the free energy will decrease as we decrease the constrained droplet mass, whereas if the vapour is supersaturated, the free energy decreases in the direction of larger droplets. There is no local minimum in the free energy as a function of the constrained droplet mass, whatever the vapour pressure: for simple systems the droplet is never in stable equilibrium with its vapour. (An exception is the case where the droplet has grown almost to fill the entire system, and the vapour pressure has been depleted considerably. In this case, the droplet is hardly a small system, nor is the vapour a bulk phase).

If we lift the constraint, the system will evolve and the constrained free energy will tell us what is likely to happen to a droplet exposed to a vapour. In non-equilibrium thermodynamics $[3,4]$, the constrained free energy is thought to play a role in the kinetics, though the details are not fully resolved. The droplet mass, or radius, is a 'relevant variable' and by fixing it and evaluating a constrained free energy, the growth and decay properties for a droplet of a particular size in a particular environment may be considered. In principle, the kinetics of droplet growth may therefore be deduced from the thermodynamics of constrained systems. Defining the most appropriate constraint, however, is a matter for debate.

Defining a molecular cluster through constraints on a microscopic level is the main subject of this paper. This is more difficult than it might sound. Clusters can quite naturally gain molecules through the capture of vapour molecules, or lose them through evaporation: a liquid cluster is only a quasi-bound structure. We have to characterise a cluster using the molecular positions and velocities and also some timescale and length scale. Such a specification will allow us to calculate a constrained free energy. Alternatively, and this is the approach taken in this paper, one could determine the rate of gain and loss of molecules directly through molecular simulation.

This sort of information is vital if we are to model nucleation, the stochastically driven formation of a stable phase (such as a droplet) from a metastable original phase (such as a supersaturated vapour). The process is often described 
in terms of cluster population dynamics. The Becker-Döring [5], or 'birth and death' equations describe the creation and loss of clusters of different sizes. The formation of large droplets is modelled as the ascent of a ladder in cluster size space, with well defined rates of up and down steps. An equivalent treatment is to consider the stochastic evolution of the size of a single cluster, with specified probabilities per unit time of growth and decay [6]. A key point in these traditional approaches is that growth and decay are assumed to be Markovian, or independent of the previous history of the cluster.

The nucleation rate turns out to depend very much on the average growth and decay rates of clusters consisting of typically only a few tens of molecules [7, 8]. Two important issues are unclear though: what is actually meant by a microscopic cluster of a particular size, and the related question of how to determine the average growth and decay rates. The way these two questions are settled fundamentally determines the nucleation model. The unresolved question of defining the positions and velocities of molecules that correspond to realisations of a quasi-bound molecular cluster has hindered the development of a microscopic theory of droplet nucleation for some time.

There have been a number of studies of this issue. Kinetic coefficients for the growth and decay of argon clusters containing between 100 and 200 atoms have been obtained through MD simulation by Shaaf et al [9]. Their simulation methodology is based on the fact that a cluster of size $N$ can be maintained at equilibrium in a confined volume if the temperature and pressure are chosen in such a way that the growth and decay rates are equal. A similar approach was also adopted by Rytkönen et al to map the phase diagram of argon clusters for various cluster sizes [10]. Zhukhovitskii has also studied a single argon cluster surrounded by vapour to obtain the size of the critical cluster as a function of supersaturation [11]. The assumption that the results obtained for small systems can be used to deduce the properties of clusters in a macroscopic vapour is central to all of these studies. This idea was tested directly by the simulations of Schaaf et al who confirmed that the evaporation coefficient is intrinsic to the cluster and independent of the surrounding vapour [9]. The dynamics of argon cluster evaporation have also been investigated using MD by Dumont et al [12]. Single clusters were equilibrated at fixed temperature and pressure, placed in a vacuum and allowed to spontaneously expand and evaporate. The evaporation timescale was found to increase dramatically as the clusters undergo evaporative cooling.

The traditional microscopic method of defining a cluster is to impose a geometric constraint on molecular positions. An example is to restrict molecules to a sphere centred on the centre of mass [13], or to impose a maximum allowable separation between the molecules (the Stillinger criterion [14]). This definition is illustrated in Figure 1(a). Atoms closer to a neighbour than a distance $R_{\mathrm{c}}$ are judged to be part of the cluster, while those beyond this radius are not. This approach is similar in spirit to the spherical droplet approach taken in continuum thermodynamics, where the droplet is defined by its spherical boundary. More sophisticated treatments, such as the $n / v$-Stillinger cluster introduced by Reiss and co-workers, are rather complex. An excellent review of recent progress in this area and the application of these ideas to nucleation theory is provided by Senger et al [15].

Such microscopic constraints can be implemented, but their realism is questionable. If we study the motion of molecules in a gas, we will find situations where molecules pass by each other without being captured. According to a geometric cluster definition we would be obliged to regard the molecules as a cluster for the short time they spend close to one another. However, the inclusion of such configurations would not be consistent with the Markovian assumption that the probability of decay is independent of the age of the cluster. Markovian decay channels are associated with fluctuation-driven escape of molecules from the cluster. In contrast, the expected remaining lifetime of a 'cluster' formed by a close passage of two molecules would decrease as time progressed. These situations should be regarded more appropriately as close encounters between separate clusters.

Clearly, then, the definition of a cluster should involve the molecular phase space and not just configuration space. We need to identify a region of phase space corresponding to a quasi-bound cluster of a certain number of molecules. Suitable integration over this region would then enable us to compute the constrained free energy of the system, if we so wished.

No consensus on phase space criteria has yet been established, though ideas have been put forward which require the cluster to be energetically bound in some sense. This must of course be a rather loose sense, since a cluster is intrinsically prone to decay. It is important to focus on the fundamental point is that a cluster will participate in birth and death-style population dynamics if its decay probability is Markovian. This is equivalent in spirit to the frequently discussed criterion that the cluster should be long-lived [16, 17]. This means that the typical lifetime, or inverse mean decay rate, is longer than typical timescales in the molecular dynamics: the decay rate is then presumed to be free of memory effects. The time evolution of a molecular configuration, and not simply the set of instantaneous positions of its component molecules, is therefore of central importance. Since the time evolution is determined by the Hamiltonian of the system, the energy of the cluster as a whole, and individual molecules in the cluster, would seem to be the key quantities to consider.

An early example of the above approach was due to Hill [18]. His cluster definition required the total energies of pairs of molecules in a cluster to be negative (in their centre of mass frame). The basic intention was to exclude situations where a molecule might be capable of leaving the cluster within the time it would take for a molecule to 
move across a typical dimension of the cluster. The definition allows some analytical progress to be made in evaluating phase space integrals, but it is not entirely satisfactory since the likelihood of molecular escape is not related just to pairwise energy contributions to the total energy. The total energy of triplets, and other groupings of molecules should also be included, though this would complicate the analysis.

Other approaches of a similar sort have been proposed. Soto and Cordero [19] have developed Hill's criterion, and Barrett [16] has performed Monte Carlo modelling of molecular configurations, excluding those which decay in molecular dynamics within a suitable period. The idea of introducing a residence time has also been discussed by both Bahadur and McClurg [20] and Pugnaloni and Vericat [21]. In these schemes, two particles are bonded at a time $t$ if they have been separated by a distance shorter than some characteristic distance $r_{c}$ for a time interval $t_{c}$. However, it is then necessary to provide a sensible estimate for the residence time $t_{c}$ as well as for the critical separation $r_{c}$.

The purpose of this paper is to propose a new cluster definition which we believe is particularly realistic. As with Barrett's approach, it is based on the idea that molecular dynamics is the only certain way of investigating whether a molecular snapshot is long-lived. Our criterion for a particle to be bound to the cluster is similar in spirit to that used in the MD simulations of Pavlov and Vorontsov-Vel'yaminov [22], whose interest was centred on phase separation in a small system. They confined 16 argon atoms within a simulation cell and monitored the relative numbers of molecules in the gas and liquid phases over a range of system energies. An atom was defined as being part of the vapour when it possessed more kinetic energy than potential energy, but only if this atom went on to collide with a wall situated $5 \sigma$ away from the centre of mass of the cluster. The authors also noted that when a particle moves from the liquid to the vapour phase its energy changes rapidly, brought about by a collision with another atom.

In our approach, we also follow the molecular dynamics until a state is reached where an evaporation has clearly occurred. We define this to be a situation where one molecule has moved so far away from the others that its interaction with them is negligible. As the system evolves towards this clear evaporation event, the cluster is considered to break at the moment when the total energy of the departing molecule becomes positive (in the centre of mass frame). The fate of the cluster is regarded as sealed once this condition is satisfied; prior to this point the cluster is quasi-bound, but afterwards it is broken. Its separation from other molecules at this instant is not directly constrained, and indeed can take a range of values, depending on its velocity. The scheme is illustrated in Figure 1(b).

In practice, the point of departure is determined by working backwards from the clear evaporation event until the departing molecules is bound. Configurations on this trajectory are excluded from consideration when calculating cluster properties. Hence we describe the definition as 'retrospective', since it is made with reference to some future clearly broken situation in the molecular dynamics trajectory. We describe the approach in detail in the next section.

We have carried out simulations employing this definition and have evaluated average decay rates for isolated Lennard-Jones clusters. In order to gather sufficient statistics, we repair the system by projecting the departing molecule back towards the cluster after every clear evaporation event. The cluster is determined to be restored by the same energy criterion that we employ to determine breakage. We then have a continuous molecular dynamics trajectory divided up into intact and broken cluster segments, as illustrated in Figure 2. The decay rate reported in section III is given by the number of evaporation events divided by the total time the system is considered to be intact.

We go on to demonstrate the relevance of molecular cluster definitions by comparing our results with the decay rates obtained with a traditional Stillinger definition based on molecular position alone. Finally, we discuss the implications of this approach with regard to more complicated systems, and how certain aspects of cluster behaviour cannot be represented with position-based schemes.

\section{MD SIMULATION PROTOCOLS AND THE REPAIR PROCEDURE}

Simulations of isolated Lennard-Jones clusters of sizes $N=10,25$ and 50 particles were performed at fixed energy using the molecular dynamics (MD) code DL_POLY [23]. The particles forming the cluster were simple Lennard-Jones atoms interacting through the pair potential

$$
U\left(r_{i j}\right)=4 \epsilon\left[\left(\frac{\sigma}{r_{i j}}\right)^{12}-\left(\frac{\sigma}{r_{i j}}\right)^{6}\right]
$$

where $\epsilon$ and $\sigma$ are the well depth and the length scale of the potential respectively, and $r_{i j}$ is the separation between atoms labelled $i$ and $j$. For an argon-like system, $\epsilon / k_{B}=119.8 \mathrm{~K}$, where $k_{B}$ is Boltzmann's constant, and $\sigma=3.405 \AA$. The cutoff radius was $50 \AA$.

For simplicity, we only wish to consider internal degrees of freedom during the dynamics, and therefore both the linear and angular momentum of these clusters are set to zero at the beginning of each run. The dynamics conserves these quantities. 
Clearly, clusters are only quasi-stable and will decay. In order to maintain the clusters at the required size, the clusters are repaired after each decay event by directing the departed atom back towards the cluster. As shown in Figure 1, the repair process reverses the direction of the escaping atom so that it rejoins the cluster. However, a simple change in sign of the velocity would provide the system with both translational and rotational energy. Therefore, we have developed a conservative repair procedure which changes the velocities of all the atoms such a way that conserves both the cluster energy and the total linear and angular momentum in a self-consistent manner. The details of this algorithm are given in the appendix. Disturbance of the system introduced by the repair process is short lived compared to the cluster lifetime since the correlation time of velocities within the cluster is very short (about 1 ps). Therefore, any memory of the details of the repair procedure is rapidly lost, and the decay rate depends only on the energy of the system and not on the way it was created. Keeping the centre of mass fixed is not a limitation of the method since the cluster lifetime does not depend on its translational energy. The lifetime is expected to depend on cluster angular momentum, though the effect is likely to be minor unless the clusters are very small, and the rotational energy large.

A simple Stillinger criterion is used to decide when a clear evaporation event has occurred and a repair is necessary. However, the critical distance $R_{\mathrm{c}}$ is chosen to be very large $\left(R_{\mathrm{c}}>5 \sigma\right)$ in comparison with the $1.5 \sigma$ that is typically used to define a geometry-based Stillinger cluster. The rationale behind this is as follows. In order to obtain the correct lifetime of each cluster, it is essential not to interfere with particles that move quite a distance away from any other atom but which would nevertheless be recaptured by the cluster at some later time without user intervention. The Stillinger radius is therefore chosen to be so large that any interaction between the escaping atom and the remaining cluster is negligible. In principle, there is no safe radius for such a criterion, but in practice, when the departing atoms is about $5 \sigma$ away from its nearest neighbour, and moving away, it is very unlikely to be recaptured.

However, the instant that such a clear signal of escape is received is not the same instant when we might regard the cluster as broken. We wish to remove any residual dependence on the loose Stillinger radius, so that in principle any value could be chosen. Having noticed a clear escape, we look back along the trajectory and regard the last moment in the lifetime of the quasi-bound cluster to be the one when the energy of the departing molecule, in the centre of mass frame, became positive. One might debate whether there is any true significance in this criterion, since the fate of the cluster will have been sealed by the deterministic dynamics at still earlier times. This point of view would only be valid if MD simulations produced completely deterministic trajectories and were not subject to the uncertainties of dynamical chaos. Therefore, we regard this energy criterion as being fairly reasonable and natural.

Recognising the precise moment of cluster breakage is important when we analyse the quasi-continuous cluster trajectory, illustrated in Figure 2, obtained by repeatedly repairing the system. In intervals around the repair events, before and after the loose Stillinger criterion is broken, there will clearly be some unbound configurations. These must be excluded to prevent a systematic overestimation of the measured lifetime. We achieve this by post-processing the trajectory. The escaping molecule is said to have become unbound at the precise moment when its kinetic energy in the centre of mass frame became greater than the magnitude of the interaction energy binding it to the other atoms within the cluster, as outlined above. Similarly, the repair procedure is said to have produced a new bound state at the exact moment when the atom becomes so close to the cluster that the magnitude of the interaction energy becomes greater than the kinetic energy. These points are indicated by asterisks in Figure 1(b). The retrospective nature of this definition is very important. During the course of the simulation, it is quite possible that atoms will move a significant distance from any neighbour and become energetically unbound from the cluster. However, without performing further MD, it is impossible to say with any certainty that the system's dynamics will not result in a new configuration which recaptures this particle before it is able to escape, unless this particle is so far away from the remaining fragment that the recapture probability is effectively zero.

There are slight complications in applying this criterion in some circumstances. If a second particle escapes in the interval between the departure and re-entry of a first particle, then the repair of this second event must be carried out before the original cluster can be considered restored. This is handled through careful post-processing. A second difficulty is that the departure of two or more atoms at the same time is actually not picked up by the loose Stillinger criterion, and the repair protocol would not then be invoked. A more elaborate check upon the configuration would recognise these situations too, but since dimer evaporation was rarely seen in our simulations, we did not regard it as a major problem.

\section{AVERAGE DECAY RATES OF ARGON CLUSTERS}

The simulation protocols described in the previous section provide a series of measurements of the lifetime of each cluster as a function of size and energy. Figure 3 shows examples of the histograms obtained when this data is binned to produce a lifetime distribution. A Markovian decay process would give rise to an exponentially distributed set of lifetimes $t$. We have therefore attempted to fit an exponential function $P(t) \propto \exp (-t / \tau)$ to these data. Figure 
4 compares the calculated inverse decay rate $\tau$ with the directly measured mean lifetimes obtained as a function of energy for each cluster size. The close agreement between these quantities indicates that cluster decay is indeed Markovian, and therefore that our simulation protocols provide cluster lifetimes that are free of memory effects.

The dependence of the mean lifetime on cluster definition is illustrated for size $N=10$ in Figure 5 . The bold line shows the true mean lifetime as a function of energy. The values shown as the dotted line are obtained instead by post-processing the trajectory using a standard $1.5 \sigma \approx 5 \AA$ Stillinger definition of cluster breakage and restoration. This geometric criterion considerably underestimates the stability of each cluster considered, and the discrepancy is more pronounced as the energy of the cluster becomes more negative. The simple geometric definition is often violated by clusters that in fact remain bound together for some considerable time. This is not surprising, since the ability of an atom to escape from the cluster depends not only upon its position relative to the remaining atoms but also upon its momentum. A $5 \AA$ Stillinger radius is most inappropriate at lower energies when atoms rarely have enough kinetic energy to become detached from the cluster, despite the fact that they may be a considerable distance from any neighbour.

This importance of including the momentum co-ordinates in any cluster definition is emphasized by Figure 6, which shows the distance between the evaporating atom and its nearest neighbour at the moment when it became unbound. The average distance is also shown: although it is fairly constant at about $4 \AA$, the variance in the distribution is very large. There is clearly no unique Stillinger radius which would correctly predict the stability of these clusters; it is necessary to consider the energies of the particles and to have some knowledge of the future behaviour of the cluster.

\section{A. Cluster Microcanonical Temperature}

Although the temperature of a macroscopic phase is a well defined thermodynamic quantity, this concept is not so easily applied to systems that contain very few atoms. This point was made clear to us by our early simulation studies in which we attempted to maintain clusters at a specified temperature using standard molecular dynamics thermostats, involving rescaling of velocities, or additional 'friction' forces. These rather unphysical procedures produced distorted dynamics and highly unstable clusters, and furthermore, we found that angular momentum was poorly conserved. Other groups have reported similar difficulties [24].

Instead we employed NVE dynamics and calculated the microcanonical temperature $T_{\omega}=\partial E / \partial S$, where $S(E)$ is the entropy of a system as a function of its energy. This is an appropriate measure of the 'hotness' of a small system at constant energy. Pearson et al [25] derived the following expression for $T_{\omega}$ for a system of $N$ particles with zero total linear and angular momentum:

$$
T_{\omega}=\frac{1}{k}\left[\left(\frac{3 N-6}{2}-1\right)\left\langle E_{k i n}^{-1}\right\rangle\right]^{-1}
$$

where $E_{k i n}$ is the internal kinetic energy of the cluster, and brackets denote time averages. We employ only intact molecular configurations to calculate the microcanonical temperature for each simulation trajectory. The repair procedure should not disturb the sampling of the microcanonical ensemble by our molecular dynamics: the procedure conserves energy and momentum, and memory of the event is lost over a timescale of a few ps, characteristic of the decay of the velocity autocorrelation function. The results are plotted in Figure 7 . The microcanonical temperature increases linearly over the energy ranges considered for the two larger clusters, but levels off at higher energies when the system is very small. The smallest clusters seem to expand as their energy becomes less negative. Therefore some of the added energy is channelled into raising the potential energy as well as into raising the kinetic energy and hence microcanonical temperature. Hence the heat capacity, or derivative of the energy with respect to temperature, rises.

It is important to note that our clusters remain liquid-like over the entire temperature range considered, even though our simulations are performed below the triple point of argon at $83.8 \mathrm{~K}$. As discussed by Rytkönen et al [10] the melting temperature of a finite system can be considerably lower than that of the bulk phase. Their simulations also reveal liquid clusters at temperatures as low as $40 \mathrm{~K}$. However, a quantitative comparison of our measured decay rates with those obtained by Shaaf et al [9] and Rytkönen et al [10] is difficult due to the larger cluster sizes considered in these studies.

We can now characterise the stability of clusters as a function of microcanonical temperature and size, as shown in Figure 8. Clusters of a given size clearly become more stable as they become colder. Furthermore, at a given temperature, the smaller clusters have a shorter lifetime. This is a typical feature of systems with a nucleation barrier. 


\section{CONCLUSIONS}

We have attempted to provide a realistic phase space definition of a quasi-bound molecular cluster. Our motivation for doing so is to establish a secure framework for calculating the relative rates of growth and decay of clusters of a particular size, as a step towards a theory of droplet nucleation. The clusters assumed to participate in the population dynamics underlying nucleation are required to be long-lived on a time scale of molecular timescales, and are characterised by size-dependent but history-independent average rates of growth and decay. This is implicit in the form taken by the birth and death equations used to describe the process.

According to theories of thermodynamic systems away from equilibrium, the rates are related to phase space integrals or constrained free energies. Equivalently, having implemented the definition into a molecular dynamics simulation, we can measure decay rates directly. After each clear decay event, when a molecule becomes extremely separated from the remaining cluster, we repair the cluster while conserving energy and linear and angular momentum. We thereby obtain a long simulation trajectory, during which a cluster breaks and is restored many times. We exclude from this trajectory the time intervals around the clear decay events during which the cluster definition is violated, to arrive at a total quasi-bound period (in a number of segments) together with a total number of decays, from which the mean decay rate, and a distribution of cluster lifetimes, can be computed.

We have studied argon clusters of 10, 25 and 50 atoms, at energies in the region of -3 to $-1 \mathrm{kcal} / \mathrm{mol}$. This range corresponds to the mean energies of clusters in a canonical distribution at temperatures of around 40-60 K. We have evaluated the microcanonical temperatures of the clusters to confirm this.

Cluster decay indeed appears to be Markovian, since the lifetimes are distributed exponentially. The mean decay rates increase with microcanonical temperature for a given size, as they should, and fall with cluster size at constant temperature. The latter is a reflection of the relative instability of small molecular clusters.

We have studied the effect of cluster definition on the decay rate. We have divided the molecular dynamics trajectories into quasi-bound and decayed segments according to a Stillinger cluster definition with a standard critical radius of $1.5 \sigma$. The decay rate so obtained is too high: clusters are judged on geometric grounds to be broken while in fact the dynamics would cause them to remain together. We have shown that the departing molecule, at the moment of cluster decay according to our definition, lies at a variety of distances from its closest neighbour. This underlines the inadequacy of a geometric definition.

Our studies have been restricted to small clusters of a simple species. However, more complicated molecules could be treated by an extension of the method. The main limitation of the scheme is that the simulation trajectory should be long enough for a few hundred cluster decays to take place. For low energy clusters, lifetimes might be too long for this to be feasible. Indirect methods might be developed to study decay in these cases, an area which is now under development.

\section{Acknowledgments}

This research was funded by the UK Engineering and Physical Sciences Research Council, under grant GR/N23400/01. We are grateful for support and advice from Prof. A.M. Stoneham.

\section{APPENDIX A: CONSERVATIVE REPAIR.}

A broken cluster needs to be repaired with minimal disturbance of the dynamics, whilst ensuring the conservation of linear and angular momentum, and energy of the cluster. This is achieved through instantaneous changes in velocities of all the molecules, not just the departing molecule. The changes can be determined as follows.

First, let us define the positions and velocities of the molecules at the moment of the repair. These are $\left\{\boldsymbol{x}_{i}, \boldsymbol{v}_{i}\right\}$ where the suffix $i$ labels the molecules in the cluster. The departing molecule is labelled $k$. The repair process is envisaged as a reversal and rescaling of the departing molecule's velocity, $\boldsymbol{v}_{k} \rightarrow-\alpha \boldsymbol{v}_{k}$ followed by a shift in the velocities of all the molecules by a constant amount, and finally the addition of rotational motion of the system about a suitable axis through the centre of mass. That is $\boldsymbol{v}_{i} \rightarrow \boldsymbol{v}_{i}+\boldsymbol{\Delta} \boldsymbol{v}_{i}$ with

$$
\boldsymbol{\Delta} \boldsymbol{v}_{i}=-\delta_{i k}(1+\alpha) \boldsymbol{v}_{i}+\boldsymbol{\Delta} \boldsymbol{v}+\boldsymbol{\omega} \times \boldsymbol{r}_{i}
$$

where $\boldsymbol{\Delta} \boldsymbol{v}$ is the constant shift, $\boldsymbol{\omega}$ is the angular velocity of the rotation, and $\boldsymbol{r}_{i}=\boldsymbol{x}_{i}-\boldsymbol{R}$ where $\boldsymbol{R}=\sum_{i=1}^{N} \boldsymbol{x}_{i} / N$ is the position of the centre of mass.

The conservation conditions allow the parameters to be determined. Firstly, the change in linear momentum is

$$
\boldsymbol{\Delta} \boldsymbol{p}=-(\alpha+1) m \boldsymbol{v}_{k}+N m \boldsymbol{\Delta} \boldsymbol{v}
$$


where $N$ is the number of molecules and $m$ is the molecular mass. Secondly, the change in angular momentum about the centre of mass is

$$
\Delta \boldsymbol{L}=-(\alpha+1) \mathcal{L}+\boldsymbol{\Lambda}
$$

where

$$
\mathcal{L}=m \boldsymbol{r}_{k} \times \boldsymbol{v}_{k}
$$

and the angular momentum due to the rotation is given by

$$
\Lambda_{j}=I_{j n} \omega_{n}
$$

with implied summation over the repeated index, where $I_{j n}$ is the moment of inertia tensor, defined as

$$
I_{j n}=\sum_{i=1}^{N} m\left(\boldsymbol{r}_{i}^{2} \delta_{j n}-r_{i}^{j} r_{i}^{n}\right)
$$

where $r_{i}^{j}$ is the $j^{\text {th }}$ Cartesian coordinate of the position $\boldsymbol{r}_{i}$.

Finally, the change in energy is

$$
\Delta E=\frac{1}{2} m \sum_{i=1}^{N}\left(\left(\boldsymbol{v}_{\boldsymbol{i}}+\boldsymbol{\Delta} \boldsymbol{v}_{i}\right)^{2}-\boldsymbol{v}_{i}^{2}\right)
$$

Some straightforward analysis shows that the conditions $\Delta p=\Delta \boldsymbol{L}=\Delta E=0$ may be satisfied with parameters:

$$
\alpha=\frac{\left(1+N^{-1}\right) A+B}{\left(1-N^{-1}\right) A-B}
$$

with

$$
A=\frac{1}{2} m \boldsymbol{v}_{k}^{2}
$$

and

$$
B=\mathcal{L}_{j} I_{j n}^{-1} \mathcal{L}_{n}
$$

together with

$$
\boldsymbol{\Delta} \boldsymbol{v}=(\alpha+1) \boldsymbol{v}_{k} / N
$$

and

$$
\omega_{j}=I_{j n}^{-1}(\alpha+1) \mathcal{L}_{n}
$$

These equations fully define the velocity shifts $\boldsymbol{\Delta} \boldsymbol{v}_{i}$ and hence the repair operation.

[1] T. L. Hill, Thermodynamics of Small Systems (Parts I and II) (Dover, 1994).

[2] H. Reiss, Methods of Thermodynamics (Dover, 1996).

[3] D. Zubarev, V. Morozov, and G. Röpke, Statistical Mechanics of Nonequilibrium Processes Vol. 1: Basic Concepts, Kinetic Theory (Berlin: Akademie, 1996).

[4] W. T. Grandy, Jr., Foundations of Statistical Mechanics Vol II: Nonequilibrium Phenomena (Reidel, 1989).

[5] R. Becker and W. Döring, Ann. Phyz. (Leipzig) 21, 719 (1935).

[6] H. Risken, The Fokker-Planck equation: methods of solution and applications, 2nd edition (Springer, 1989).

[7] D. Kashchiev, Nucleation: Basic theory with Applications (Butterworth Heinemann, 2000).

[8] I. J. Ford, Phys. Rev. E 56, 5615 (1997).

[9] P. Schaaf, B. Senger, J. C. Voegel, R. K. Bowles, and H. Reiss, J. Chem. Phys. 114, 8091 (2001).

[10] A. Rytkönen, S. Valkealahti, and M. Manninen, J. Chem. Phys. 108, 5826 (1998). 
[11] D. I. Zhukhovitskii, J. Chem. Phys. 103, 9401 (1995).

[12] R. S. Dumont, S. Jain, and A. G. Basile, J. Chem. Phys. 102, 4227 (1995).

[13] H. Reiss, J. L. Katz, and E. R. Cohen, J. Chem. Phys. 48, 5553 (1968).

[14] F. H. Stillinger, J. Chem. Phys. 38, 1486 (1963).

[15] B. Senger, P. Schaaf, D. S. Corti, R. Bowles, J. C. Voegel, and H. Reiss, J. Chem. Phys. 110, 6421 (1999).

[16] J. C. Barrett, J. Chem. Phys. 116, 8856 (2002).

[17] A. A. Lushnikov and M. Kulmala, Phys. Rev. E 58, 3157 (1998).

[18] T. L. Hill, Statistical Mechanics: principles and selected applications (McGraw-Hill, 1956).

[19] R. Soto and P. Cordero, J. Chem. Phys. 110, 7316 (1999).

[20] R. Bahadur and R. B. McClurg, J. Phys. Chem. 105, 11893 (2001).

[21] L. A. Pugnaloni and F. Vericat, J. Chem. Phys. 116, 1097 (2002).

[22] V. A. Pavlov and P. N. Vorontsov-Vel'yaminov, High Temperature 15, 1165 (1977)

[23] DL-POLY is a package of molecular simulation routines, written by W. Smith and T.R. Forester, copyright The Council for the Central Laboratory of the Research Councils, Daresbury Laboratory, nr. Warrington (1996).

[24] H. Vehkamäki, private communication.

[25] E. M. Pearson, T. Halicioglu, and W. A. Tiller, Phys. Rev. A 32, 3030 (1985). 


\section{Figures}

1. Cluster definitions. In sketch (a), a molecule is considered to belong to the cluster if it lies within a distance $R_{\mathrm{c}}$ of another molecule in the cluster. In sketch (b), a representation of our model, the criterion is more complicated, and involves molecular dynamics. In a decay event, a molecule follows a trajectory taking it far away from the other molecules, and it leaves the cluster at the point on this trajectory when its energy becomes positive. When returned to the cluster, it joins when its energy becomes negative.

2. The continuous molecular dynamics trajectory for the system consists of a sequence of repair procedures at times when the loose Stillinger criterion is broken. Around these events, parts of the trajectory are excluded from the calculation of cluster properties, since the cluster definition described in Figure 1(b) is not satisfied.

3. Example histograms showing the distribution of lifetimes obtained for $N=10$ (top), $N=25$ (middle) and $N=50$ (bottom) clusters. The histograms shown illustrate the decay of the lowest energy (left) and the highest energy (right) state studied for each cluster size.

4. The mean lifetimes (full line) and calculated inverse decay rates $\tau$ (dotted line) of clusters with $N=10$ (top), $N=25$ (middle) and $N=50$ (bottom).

5. The effect of the cluster definition on the measured lifetime of the $N=10$ cluster. Results using a $1.5 \sigma$ Stillinger criterion are shown by the dotted line; those obtained using our dynamical cluster definition are shown by the full line.

6. Distributions and means of the distance between the escaping atom and its nearest neighbour at the instant of decay for the $N=10$ cluster.

7. The microcanonical temperature as a function of energy for the $N=10$ (full line), the $N=25$ (dotted line) and the $N=50$ clusters (dashed line).

8. The mean lifetime as a function of microcanonical temperature for the $N=10$ (full line), the $N=25$ (dotted line) and the $N=50$ clusters (dashed line). 

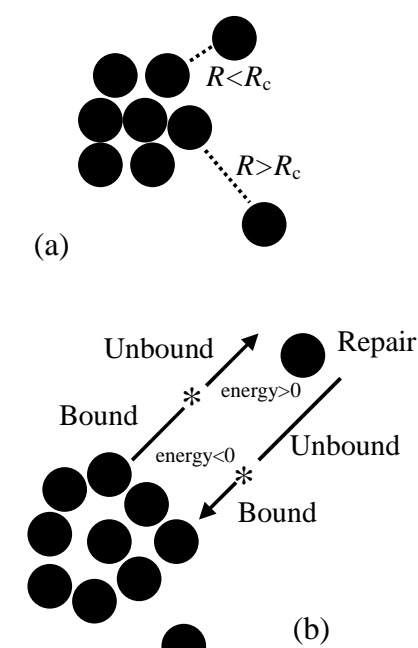

FIG. 1: 


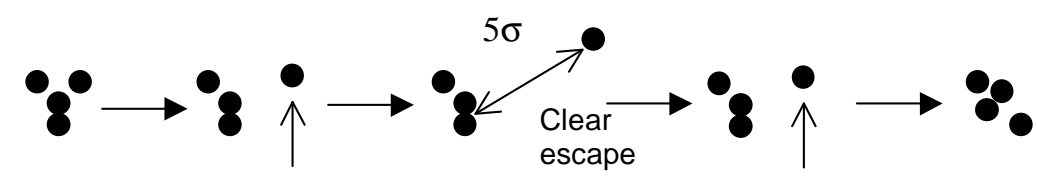

Energy becomes +ve velocity Energy becomes -ve reversed

\begin{tabular}{c|c|c}
\hline intact & broken & intact \\
\hline time $\longrightarrow$
\end{tabular}

FIG. 2: 

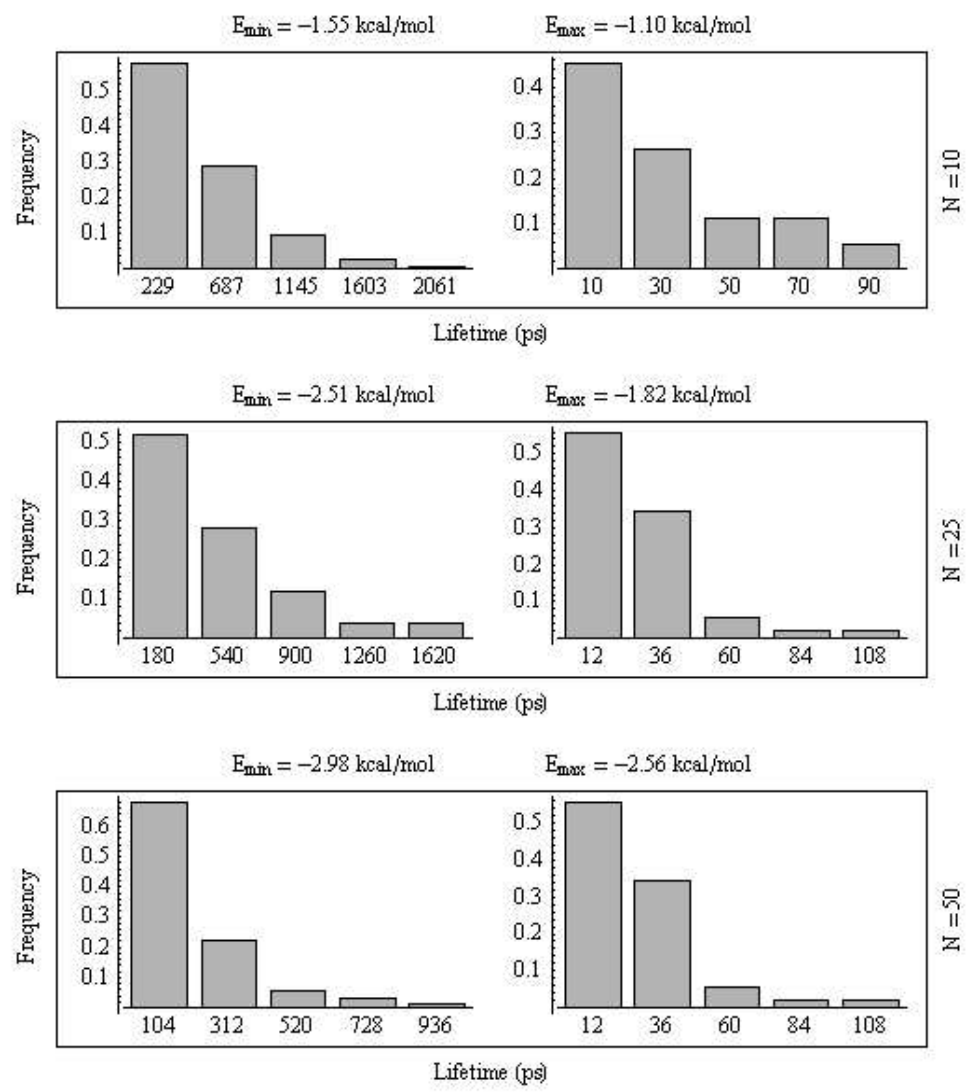

FIG. 3: 

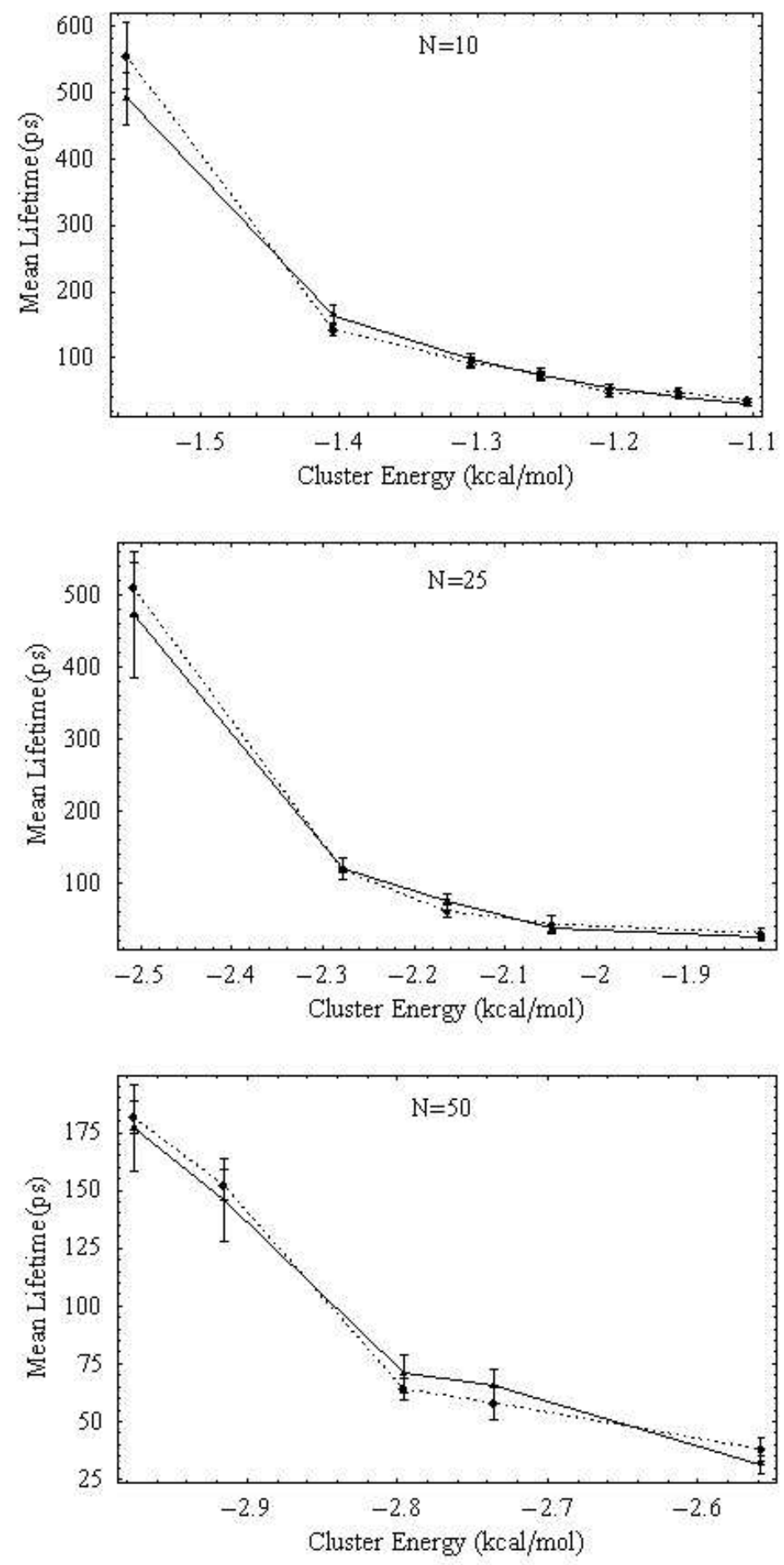

FIG. 4: 


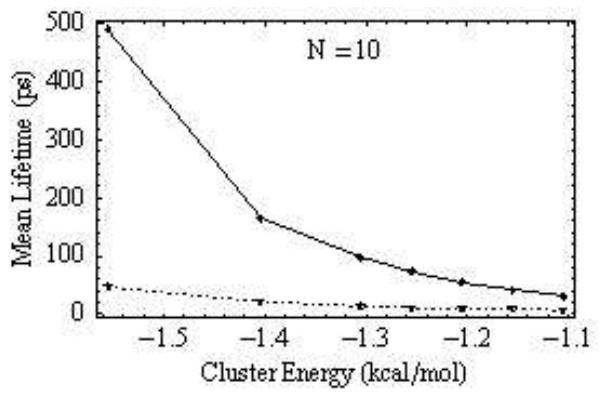

FIG. 5: 


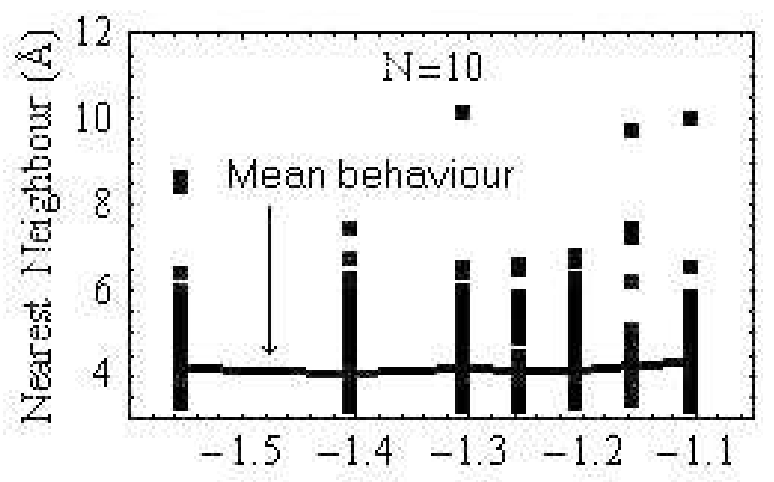

Cluster Energy (kcal/mol)

FIG. 6: 


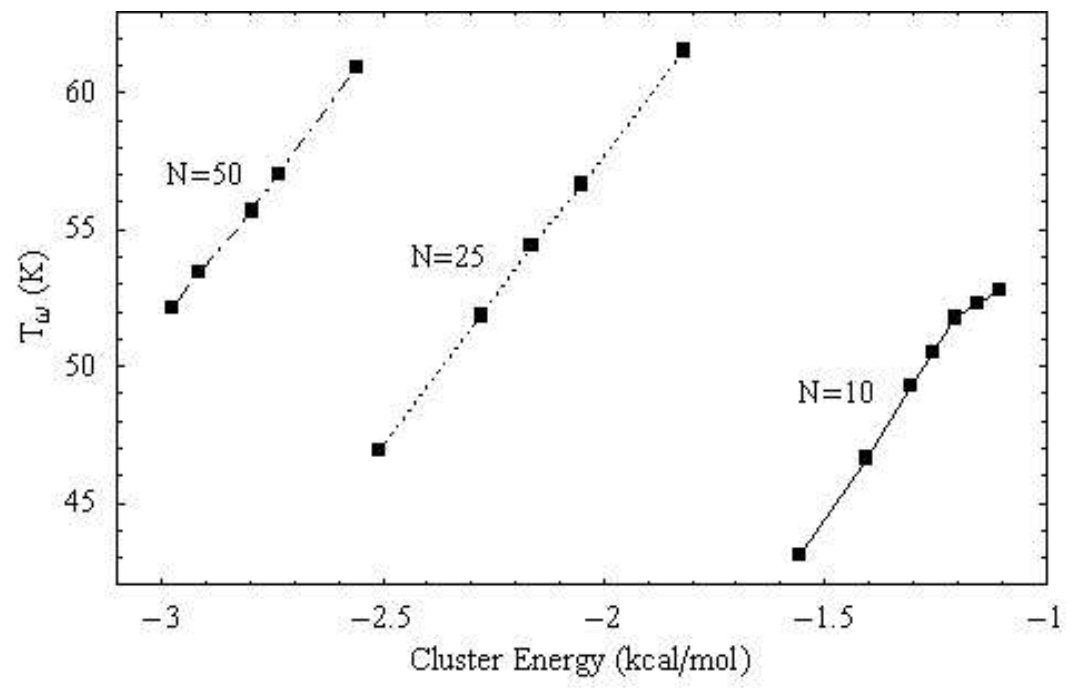

FIG. 7: 


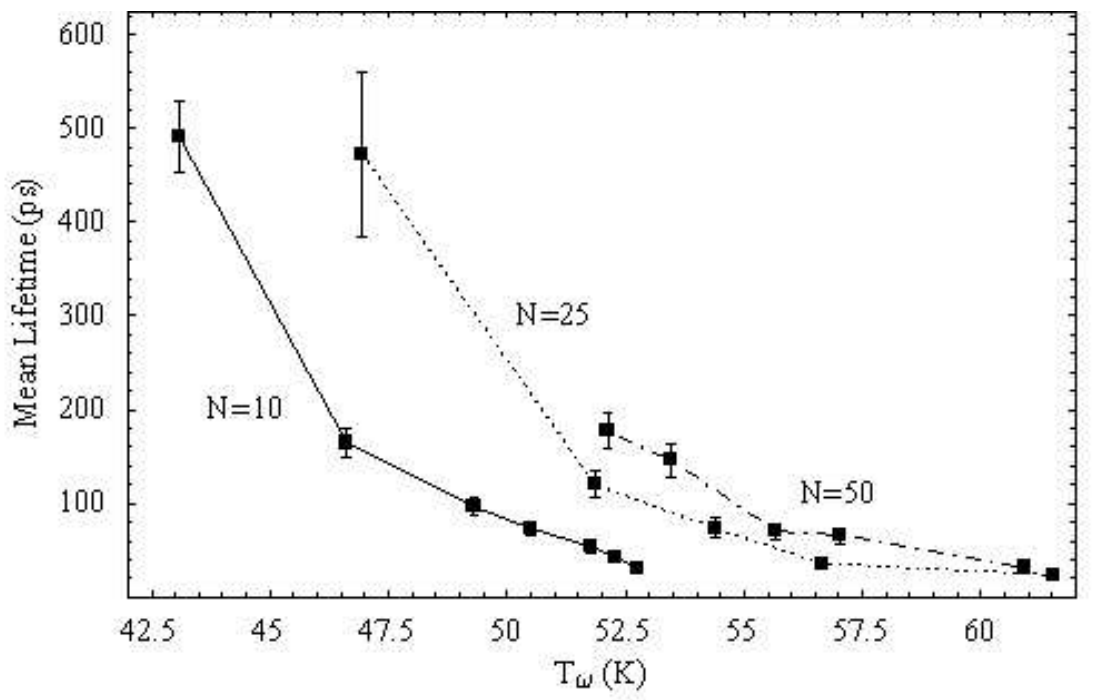

FIG. 8: 